d'ouverture a été construit et installé à l'Observatoire de Paris. L'appareil est en cours de réglage au moment où l'on écrit cette note.

Tout ce qui précède se rapporte à la détermination des ascensions droites. Des dispositions spéciales sont à l'étude en vue de l'observation du Soleil.

8. Quant aux déclinaisons, il semble hautement désirable de les déterminer sans l'aide d'un cercle gradué. L'instrument des passages à réflexion peut être adapté à l'observation des passages au premier vertical; il conserve alors les avantages qu'il possède sur l'instrument méridien classique. Mais, à Paris, cette méthode ne s'applique avec une bonne précision qu'aux déclinaisons comprises entre $29^{\circ}$ et $48^{\circ} 50^{\prime}$.

L'auteur a décrit en 1948 un astrolabe à prisme impersonnel d'un type nouveau, (Comptes Rendus de l'Académie des Sciences, Paris, 227, 320), qui permettrait d'étendre le champ des observations. Un modèle de cet astrolabe, de $6 \mathrm{~cm}$ d'ouverture, est actuellement en service à l'Observatoire de Paris. L'écart quadratique d'un passage d'étoile à la distance zénithale de $30^{\circ}$ est habituellement de 0 ". 3 , mais il s'abaisse à o".2 quand les conditions atmosphériques sont favorables. Divers perfectionnements mécaniques permettront sans doute d'améliorer encore les observations. D'autre part, deux instruments de plus grande ouverture sont à l'étude.

Un tel instrument peut servir d'instrument fondamental pour les étoiles dont la déclinaison est comprise, pour la latitude de Paris, entre $+\mathrm{I} 8^{\circ} 5 \mathrm{O}^{\prime}$ et $+78^{\circ} 5^{\prime}$. Toutefois, les déclinaisons des étoiles zénithales ne seraient pas déterminées avec précision.

A Paris, l'emploi combiné d'un instrument dans le premier vertical et d'un astrolabe fournirait les déclinaisons des étoiles d'une première zone de déclinaison, comprise entre $+18^{\circ} 5^{\prime}$ et $+48^{\circ} 50^{\prime}$, et d'une seconde zone s'étendant approximativement de $+60^{\circ}$ à $+78^{\circ} 50^{\prime}$, laissant des lacunes faciles à combler grâce à la coopération d'autres observatoires disposant d'un matériel analogue, ou, plus simplement, d'astrolabes du type nouveau.

\title{
PHOTOGRAPHIC DETERMINATION OF THE MOON'S POSITION, AND APPLICATIONS TO THE MEASURE OF TIME, ROTATION OF THE EARTH, AND GEODESY
}

\author{
BY WM. MARKOWITZ
}

\begin{abstract}
A dual-rate moon position camera is used to hold the moon and stars fixed during a simultaneous time exposure. The coordinates of the moon with respect to the stars are found with greater accuracy than has been previously possible. Also, the moon may be observed over a greater orbital arc. These advantages combine to make the photographic technique a powerful one in the solution of several astronomical and geodetic problems.
\end{abstract}

I. Introduction. Of all celestial bodies the moon has the most rapid motion about the earth and the largest parallax. There has been increased emphasis in recent years, as a consequence, on the use of the moon in the solution of various astronomical and geodetic problems. Field techniques of high accuracy have been developed, which make use of observations of solar eclipses and of occultations at carefully selected stations. Two special projects, whose results will be needed in making a definitive reduction of lunar observations, are nearly complete. These projects, supported in part by ONR, are the charting of the moon's limb by Dr. C. B. Watts, and the joint computation of a more precise lunar ephemeris by the U. S. Nautical Almanac Office, the British Nautical Almanac Office, and the Watson Scientific Computing Laboratory.

The classical methods of observing the moon are subject to many restrictions regarding the place and time of observation, and the phase of the moon. Hence astronomers have attempted for a long time to bring the flexibility and accuracy of the photographic method to bear on the problem of finding the moon's position. Formidable difficulties are encountered, however, because of the motion, brilliance, and size of the moon, and only one such program, to my knowledge, has hitherto been carried out. Plates were taken at Harvard, in I9II-I7, by A. S. King and were reduced at Princeton by Prof. H. N. Russell and colleagues. ${ }^{1}$ A snapshot of the moon was taken 
at the middle of a Io-minute exposure on the stars. Although no correction was applied to the clock drive during the long exposure, results of comparatively high accuracy were obtained by Prof. Russell.

In the new photographic technique to be described, the moon and surrounding stars are exposed simultaneously for about 20 seconds, and the moon is held fixed relative to the stars during the exposure. Use is made of a dual-rate moon position camera, which can be attached to either a visual or a photographic refractor of about 8 or more inches aperture and of moderate focal length, preferably 80 to 240 inches.

A dual-rate camera was attached to the I2inch visual refractor of the U. S. Naval Observatory, and a regular program of observing was started on 2 June 1952. It has been found that good plates can be obtained even under fair conditions, for example, at full moon, with light haze present, and with the moon at a zenith distance of $67^{\circ}$. The internal probable errors for one plate are 0 ". $\mathrm{I} 5$ in right ascension and 0 ". 10 in declination.

The position of the moon is based on measures made on a large number of points on the bright limb. Hence the effect of limb irregularities on the derived position is much less than in the methods which involve but a single point. No corrections for limb irregularities have been made thus far. They will be applied, however, when the corrections by Watts become available.

2. Principles of operation. The principal features of the camera are shown in the figure. Precise guiding is needed on both stars and moon, and the telescope clock drive is not used. The synchronous motor and micrometer, shown at the left, drive the moving plate carriage at the sidereal rate. The moon's image is intercepted by a dark filter, of transmission factor 0.00I, which is a plane-parallel glass plate $1.8 \mathrm{~mm}$ thick. The motor and micrometer shown at the top tilt the filter during the exposure, thereby shifting the image of the moon. The rate of tilt is adjusted so as to hold the moon fixed relative to the stars.

Three settings are made on the camera when observing: for the sidereal rate corresponding to the moon's declination, for the moon's speed, and for the position angle of the axis of tilt. The computations and mechanical settings are easily made.

A light yellow filter having a hole at the center cuts out the blue rays of the stars. An electrical contact, shown at the top, is set to record on a chronograph the instant when the two filters are parallel. At this moment the filters produce no relative shift of the moon and stars. Hence, this instant defines the epoch of observation. The adjustment for timing, which is easily made by examining reflected images, remains fixed. If the camera is reversed $180^{\circ}$ between exposures any error in timing is eliminated.

A light fixed to the camera-body generates a trail about $6 \mathrm{~mm}$ long during the exposure. This trail allows the plate to be oriented rapidly in the measuring engine and serves to check the orientation of the camera.

3. Applications. (a) The measure of time. The relation between Ephemeris Time (uniform) and Universal Time (non-uniform) is given by

$$
\begin{aligned}
& \Delta T=\text { E.T. }-\mathrm{U} . \mathrm{T} . \\
& \Delta T=\text { I.82 I } \Delta L
\end{aligned}
$$

where $\Delta L$ is the observed correction to the tabular mean longitude of the moon given by the new lunar ephemeris. $\Delta T$ is in seconds of time when $\Delta L$ is in seconds of arc.

Observations made at one station during one lunation should determine $\Delta T$ with a probable error of $\mathrm{O}_{\mathrm{s}}^{\mathrm{s}}$, and if made over a year should determine absolute frequency to about 3 parts in $10^{9}$. If similar observations were to be made at several observatories it should be possible to determine absolute frequency to about one part in $10^{9}$.

It may be noted that as of this date there is no atomic frequency standard in day-to-day operation with which astronomical results can be compared. Isolated runs made with the cesium beam at the National Bureau of Standards would appear to indicate an accuracy of one part in $10^{8}$. There is little doubt that an accuracy of a part in $10^{9}$ will be reached, but we do not know when. In the meantime, progress on atomic frequency standards would be enhanced if astronomers could furnish uniform frequency.

(b) Changes in the rate of rotation of the earth. It is known that irregular changes in rate which may amount to one part in $2 \times 10^{7}$ occur; these are believed to be due to geophysical causes. It is of interest that a noticeable speeding up in rate occurred during 1953. A comparison of the Washington and Richmond photographic zenithtube observations with the extrapolated performance of quartz-crystal clocks shows that the change in rate was not sudden but that there was a series of sudden changes in acceleration, which 


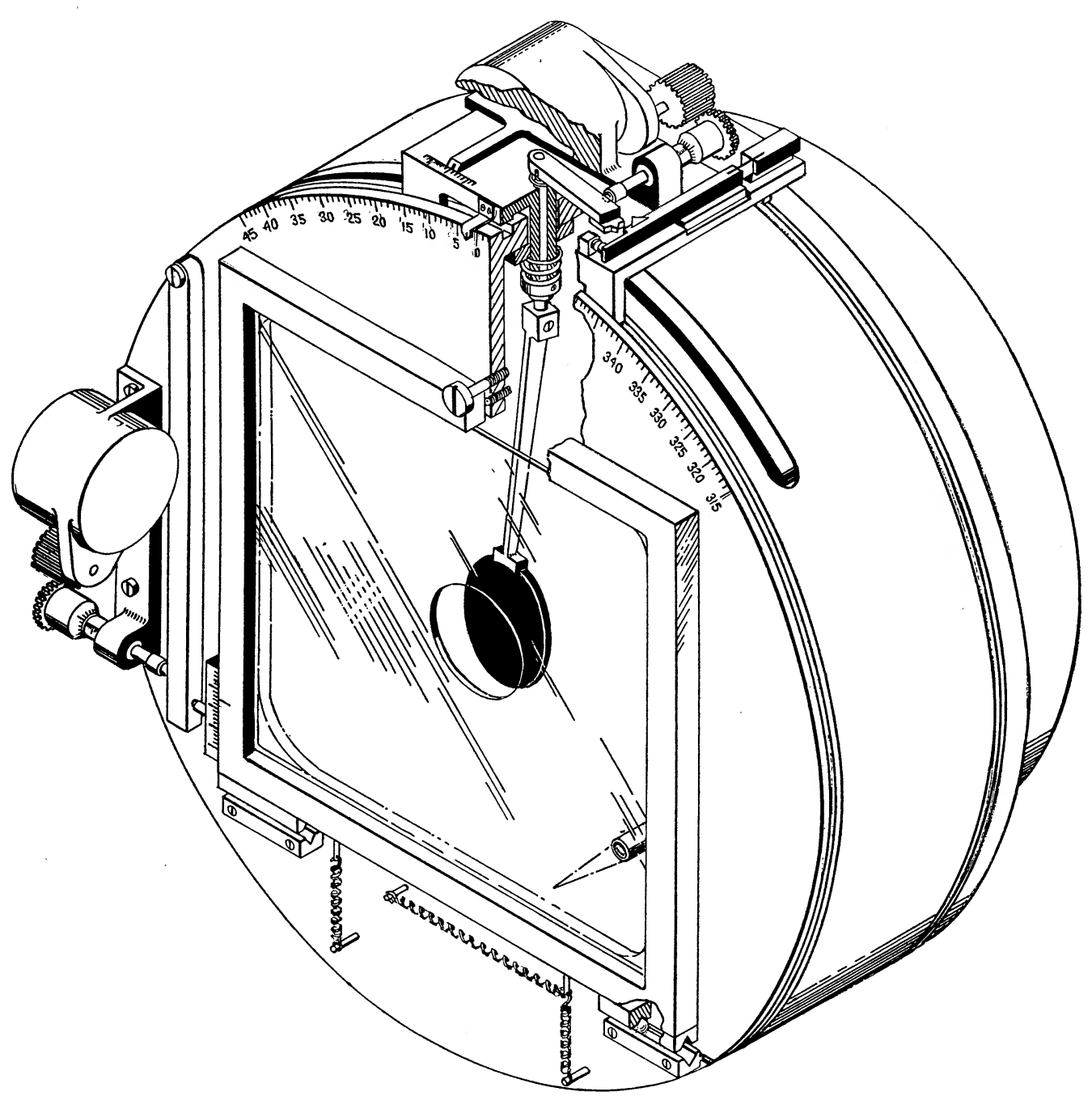

Figure. Dual-rate moon position camera, schematic view.

occurred about a month or two apart. The value of the attendant change in rate which was found cannot be regarded as definitive because of the uncertainty in the clock extrapolation. Definitive values, however, can be obtained from observations of the moon. Should several observatories photograph the moon regularly, it would be possible to detect small changes in rate which might be correlated with geophysical phenomena.

(c) Geodesy. While the moon has of course been used in the solution of isolated geodetic problems I would like to discuss what may be called the general problem. This means a determination of corrections to the adopted constants of the moon's orbit including the parallax; to the size and shape of the earth; and to the deflection of the vertical and the elevation above the reference spheroid for each of the observing stations. In order that the unknowns may be well separated it is necessary to have a large number of observations which must be taken under widely different astronomical conditions. 
The field methods, although accurate, do not meet these requirements. The beautiful eclipse path in 1954 which connects North America, Scandinavia, eastern Europe and Iran will not be duplicated for several hundred years. The occultation method, I am informed, cannot even be used so far north of the tropics. Eclipses can occur only when the moon is new, and suitable occultations only when the moon is within three days of first quarter. It would appear, therefore, that these methods cannot be used to solve the general problem. They can, however, make significant contributions to parts of the problem and for that reason they should be continued.

The photographic method has few restrictions; observations can be made at any phase except within a few days of new moon, and over a large range in hour angle and zenith distance. At Washington, for example, the parallax factor may vary from 0.0 to 0.9 in right ascension and from 0.2 to 0.9 in declination. This method may be used at fixed observatories with telescopes already in existence, especially the astrographs used for the Carte du Ciel. If about ten or fifteen observatories suitably spaced around the world were to engage in a cooperative program it should be possible to obtain a solution to the general problem in about a year or so of intensive observation.

(d) The motion of the moon. Although the theory of the motion of the moon developed by E. W. Brown is considered to be sufficiently exact for all necessary purposes, the constants entering into the theory are subject to improvement. Brouwer and Watts have derived certain corrections through discussion of meridian transits and occultations. ${ }^{2}$ The use of the photographic method, however, should provide a more accurate set of corrections because of the larger orbital arc through which the moon can be observed and because of the greater freedom from systematic and accidental errors.

4. Systematic errors. A comparison of the results from night to night indicates an external probable error in either coordinate of about o". I5, a value which may be reduced when limb corrections can be applied. Accepting this value, however, the effective probable error of the moon's position derived from observation on 100 nights would be o".o 5 if there were no systematic errors. Such errors, if large, would seriously affect the geodetic work. Thus, an unknown systematic error in the measuring engine which causes a systematic error of one micron at the center of the plate would cause a systematic error in position of o".045 for the Washington plates.

The systematic errors can be greatly reduced by use of certain standard procedures. These include: (a) investigation of the measuring engine, (b) reading alternate plates in the direct and reversed directions, (c) photographing standard star fields at various hour angles, and (d) reversing the camera $180^{\circ}$ between exposures.

If these steps are followed I think that the error could be held to o".02. This corresponds to an uncertainty in geodetic distance of $\mathbf{I} 20$ feet ( 37 meters). The uncertainty could be further reduced by adjusting a complete global net. The probable error of the derived deflection of the vertical in either coordinate should be about $\mathrm{I}^{\prime \prime}$.

5. Measurement and reduction. A double-screw measuring engine is used to obtain the $x, y$ coordinates of about io stars and about 30 or 40 points on the moon's bright limb. A few preliminary measures are made to determine the center and radius of a provisional circle which fits the bright limb. The coordinates of points $6^{\circ}$ apart on this circle are then computed. In measuring the moon one screw is set on a computed coordinate, say $y$, and the other screw moves the cross threads up to the limb in $x$. We thereby obtain a differential correction to the radius of the form $d r=(x / r) d x$ since $d y=0$. The $d r$ 's are combined in groups to give about Io or 12 normal points.

It is proposed to simplify the measuring process through the use of a coordinate measuring machine which has an accurate rotating stage. The $d r$ 's could then be measured without preliminary computation.

The determination of the plate constants allows corrections to be made for all first-order terms, including first-order differential refraction. These corrections are applied to the normal points, and the position of the center of the circle of best fit is found by least squares.

The star positions, taken from the Yale catalogs, are brought up with proper motion to the epoch of date, but referred to the equinox of I950.0. The usual reduction to change to the equinox of date is made and the corrections for parallax in the two coordinates are applied. A transfer is made to the system of the New Zodiacal Catalog, which is virtually on $\mathrm{FK}_{3}$, by applying corrections NZC-Yale obtained from about a dozen stars near the moon's position. 
The final position may then be compared with the ephemeris of the moon.

The corrections for limb irregularities will be made at a later date.

It may be noted that in this method a semidiameter of the moon is not assumed. The derived position of the moon's center is thus independent of personal equation in setting on the limb. The question of whether it would be profitable to assume a semi-diameter, especially when the phase is small, has not yet been thoroughly investigated.

6. Instrumental and operational data. A brief description of the apparatus and of the method of operation at Washington, which will now be given, may be useful in setting up a similar program elsewhere. A more detailed description of the program will be published in the future. Additional information, in the meantime, will gladly be given if desired.

(a) Telescope. This is a $\mathrm{I} 2$-inch visual refractor of $\mathbf{I} 8 \mathrm{o}$ inches focal length made by Clark. The stars measured usually lie within $50^{\prime}$ of the center. Faint coma is visible on the outer images, but this causes no trouble in measuring. The field is not flat, but this is remedied somewhat by having the star filter slightly thicker than the moon filter. No change in focus is made for temperature variation. The definition on the moon and stars is good.

The finder contains a plastic reticle marked with several concentric circles. When the moon's bright limb coincides with the proper circle the drive motors are turned on. The shutter is then opened for the required exposure.

(b) Camera. In designing the dual-rate camera two considerations were kept in mind, simplicity in construction compatible with the required accuracy and simplicity in operation. Commercial micrometers and motors were used, and ordinary machining operations were sufficient for the construction.

Observations may be made rapidly in the dark. Four plates are taken in five minutes, and only about fifteen minutes need be spent in the dome.

(c) Plates. The plates used are Eastman II-G when the moon is bright and I03-G when faint. The plates are sensitive up to $5700 \mathrm{~A}$ and the filter for the stars cuts off sharply at 5000A. The filter is Schott GG-I4A, size I70 X I70 mm. The plate size is $7 \times 7$ inches.

The plates are carried in covered aluminum sheaths. During the exposure the sheath presses the emulsion surface against a metal frame on the carriage, which defines the focal plane.

(d) Exposures. The exposures range from I $2^{\mathbf{s}}$ with the II-G plates at full moon to about $24^{\mathrm{s}}$ with the I03-G plates at quarter. Four plates are taken at one time and two of these are measured. There is little more work involved in reducing two as compared to one, while little would be gained in measuring more than two. Unless the moon is in a very poor region enough Yale stars can generally be found to reduce the plates.

(e) Future improvements. Several changes are contemplated. It is intended to have more nearly automatic operation and to allow rapid reversal of the camera. If a simple means of darkening the Schott filter near its central aperture can be found it will help reduce sky fog near the moon, and thus make it easier to measure the plates by projection.

7. World wide program. The forthcoming International Geophysical Year, I957-58, will involve a number of operations of geophysical interest. At a meeting held in Brussels in July I953, a resolution was adopted recommending the inclusion of a world-wide program of photographic moon observations. It is planned to observe at about fifteen stations and to measure at about four. The Office of Naval Research has offered to support the major part of the cost of construction of the cameras and measuring instruments. About ten observatories located in various parts of the world have already expressed an interest in participating in the program. The coverage can be improved in certain parts of the world, however, and inquiries from other observatories will be welcome.

\section{REFERENCES}

I. Ann. Harv. Coll. Obs. 72, 1, І9113; 76, 127, г916.

2. A. J. 52, 169, 1947 .

U. S. Naval Observatory,
Washington, D. C. 1953 September. 